\title{
Research on the Performance of the Offshore-Platform Air Filter
}

\author{
Nan Ye ${ }^{1}$, Tao Sun ${ }^{1^{*}}$, Chang-jiang Sun ${ }^{2}$ and Zheng-wei $\mathrm{Ma}^{3}$ \\ 1. College of Power and Energy Engineering, Harbin Engineering University, Harbin 150001, China \\ 2. Military agent's room, Dalian Shipbuilding industry co., Ltd, Dalian, 160001, China \\ 3. 703th Research Institute of China Shipbuilding Industry Corporation, Harbin, 150078, China \\ *Corresponding author Email: heu_jf@163.com
}

Keywords: porous medium; air filter; experiment; CFD

\begin{abstract}
Air-intake filter in the ventilation system of offshore platform, is normally composed of the inertial stage and the multi-layer gauze stage, which plays an important role in providing sufficient and clean air. Numerical simulation and experimental research were carried on to study the flow field and resistance characteristics of the air-intake filter. Flow in the inertial stage was regarded as a 2D case, and the multi-layer gauze stage was simplified to an orthogonal array type. The quadratic polynomial of the resistance with velocity, and the permeability $\alpha$ and the pressure jump coefficient $\mathrm{C}_{2}$ of the composite stages were obtained on the basis of certain simplifications. By one source term added into the momentum equation, the flowing characteristics of the filter compounding the inertial stage and the gauze stage were computed based on the porous medium model. The simulating results of the total pressure loss are validated by the designed wind-tunnel experiment satisfactorily. The method was proved to be useful on the design and optimization of the air-intake filter on offshore platforms and other occasions.
\end{abstract}

\section{Introduction}

Ventilation on the offshore platform is essential both to the working place and the living area. Besides reducing the concentration of the flammable, toxic, explosive gas and the steam or dust, ventilation plays an important role in providing sufficient and clean air to the combustion of the gas turbine, diesel engine and heating furnace etc. The purpose also lies in guaranteeing a reasonable distribution of pressure on the platform. Nevertheless, the atmospheric environment makes it almost impossible for the offshore platform to obtain dry and clean air directly. Studies have shown that the salinity and seawater droplet always exist in the oceanic atmosphere in the form of aerosol (Brekke et al., 2010). Therefore, air-intake filter, which aims at ensuring the safe operation, is always affiliated to the ventilation system of the platform.

The filtration part of the filter is normally composed of the inertial stage and the multi-layer gauze stage. Inertial stage is widely used for the less pressure loss and higher separation efficiency of liquid drop with diameters of 10-40 $\mu \mathrm{m}$ (Chiara Galletti et al., 2008). The gas pass through the inertial stage was described by Reynolds Averaged Navier-Stokes equations (Luan Yigang et al., 2010) closed with k- $\varepsilon$ model proposed by Rodi (1980). One simply algorithm, proposed by Patankar and Spalding was used to solve the equations (B.E.Launder et al.,1972; S.V.Patanker et al., 1972). Early design of the multi-layer gauze stage was principally based on Souders-Brown equation (Souders et al., 1961) and semi-empirical formula found on it (York et al., 1954). Sun Haiou et al. (2000) suggested a new CFD model for the multi-layer gauze stage that crossed knitted wire meshes are simplified to orthogonal wire mesh holes (Sun Haiou et al., 2000).

Meticulous experiments were carried out to research the effects of the droplet diameter, the inlet velocity and the length of the flowing path on the cyclone separator the inertial stage and the multi-layer gauze stage (Elisabetta et al., 2001). And the resistance characteristic of the separator was investigated, which shows the total pressure loss is a significant indicator to evaluate its performance (Sun Haiou et al., 2002; Rafee, R. et al., 2010).

In this paper, flow field characteristics of the offshore platform air filter was studied through the numerical simulation and experimental methods. 


\section{Model Simplifications}

The filtration part of the offshore-platform air filter is composed of the inertial stage and the multi-layer gauze stage, whose performance has respect to that of the filter closely. Considering the complex constructions of the above two stages, the porous medium model is employed to simulate the flowing characteristics of the compound model based on the following simplifications.

The porous medium model employed in the study is based on the simplifications of the inertial stage and the multi-layer gauze stage. Meanwhile the grid number of CFD simulation is reduced and its quality is improved.

\section{Simplification of the Inertial Stage.}

The height of the inertial stage is customarily 20 times more than the width, and the interior flowing velocity is low. The upper and lower parts of the stage have limited influence on the whole stage. In view of this, the flow characteristics of filter can be represented by a certain horizontal section. With little errors, the 2D model would simplify the calculation. The simplified inertial stage is shown in figure 2, and a certain length extension is added at the front and rear ends of the inertial stage respectively.

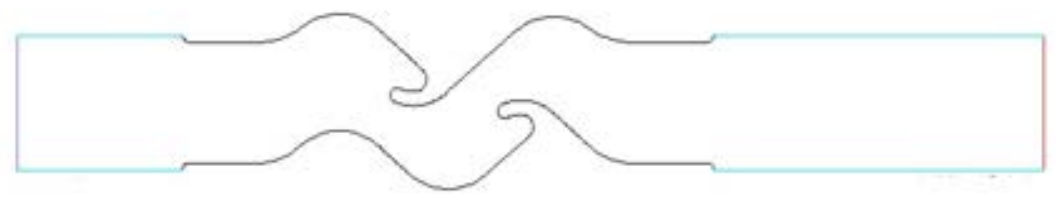

Fig.2 Mode of the simplified inertial stage

The inertial stage is composed of zigzag blade with a hook shaped drain tank. In the light of the flow periodicity, one single flow passage between two plates is selected as the computational domain and edges representing the plates wall are defined as periodical boundary. The width of the inertial stage is $11.2 \mathrm{~mm}$.

\section{Simplification of the Multi-Layer Gauze Stage.}

The multi-layer gauze stage of the air filter is constituted of 120 layers of surrounded wire meshes and each layer is distributed randomly. Referring to the two dimensions computational model suggested by Sun Haiou and considering the huge difference in the orders of magnitude of the gauze diameter and its mesh size, crossed knitted wire meshes are simplified to parallel ones and ring shape filter element is simplified into cuboids shape filter element. Both of them share the same thick, which is the difference between the inside and outside radiuses. The simplified partial model of 12 layers is indicated in Figure 3.

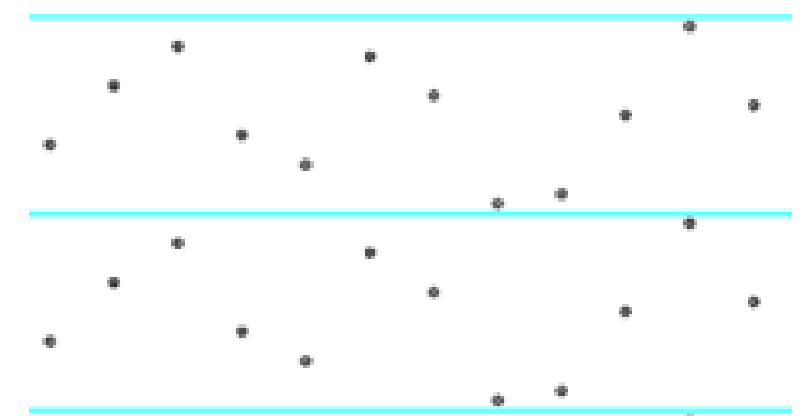

Fig.3 Mode of the simplified multi-layer gauze stage

Edges are set as periodic boundary besides the inlet edge and outlet edge. The mesh size is $3 \mathrm{~mm} \times 4 \mathrm{~mm}$, the mesh diameter is $0.12 \mathrm{~mm}$ and the spacing of adjoining mesh is $0.5 \mathrm{~mm}$.

Simplification of the Air Filter.

The eight faces, from which the oceanic air empties into and through the inertial stage and the multi-layer gauze stage, are axially symmetrical. The air runs out of the cylindrical channel under the rectifying action of the filter. Periodical boundary is also adopted, the whole and the eighth model of 
the filter is illustrated in figure 4.

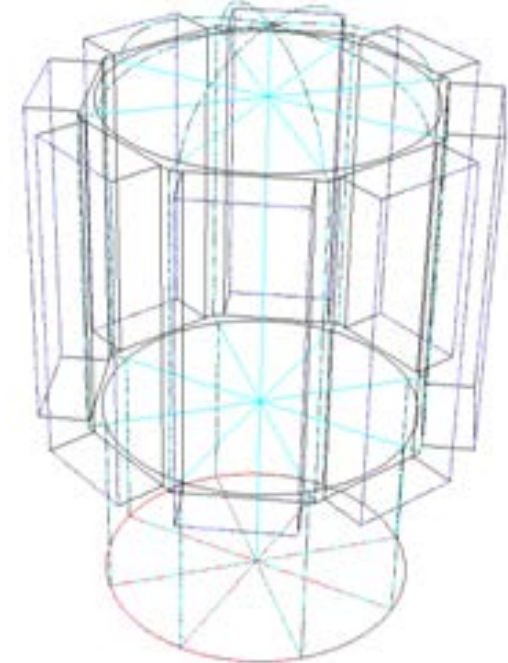

(a) Simplified filter model(whole)

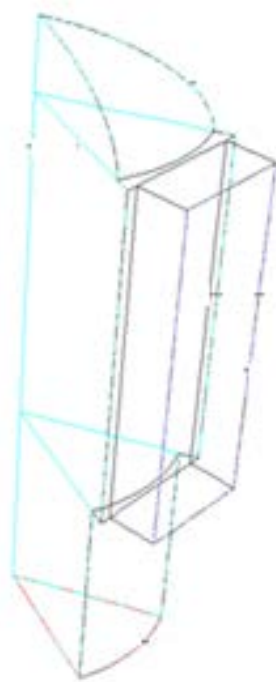

(b) Simplified filter model(eighth)

Fig.4 Mode of the simplified air filter

Periodic boundaries, displayed with light green lines in the Figure 2-4, were used in the CFD simulations of the inertial stage, the gauze stage and the air filter. Besides, the inlet and outlet boundaries are shown with purple and red lines respectively. All the quadrilateral grids of the filter model were generated in Gambit.

\section{Boundary Conditions}

The boundary conditions for numerical simulation of the models are as follows:

(1)Inlet: Standard atmospheric pressure, $\mathrm{P}=101325 \mathrm{~Pa}$ (gauge pressure: 101325Pa); Temperature, $\mathrm{T}=300 \mathrm{~K}$.

(2)Outlet: Outlet static pressure, varying to correspond with the different inlet velocity.

(3)Steady wall: Heat isolated; No slip.

(4)Outlet of the compound stage (for the air filter model only): Porous step.

\section{Simulation and Experiment Results}

\section{Resistance Characteristic of the Compound Stage.}

The inertial stage and the multi-layer gauze stage constitute the compound filtration part of the offshore-platform air filter. Figure 5 and 6 exhibit the total pressure distributions of the inertial stage and the multi-layer gauze stage. The structures of the two stages lead to the pressure loss of the inlet gas fluid. Furthermore, drain hooks of the inertial stage would cause the sudden change of the velocity, and the gauze stage has an effect on the control of the flowing turbulence.

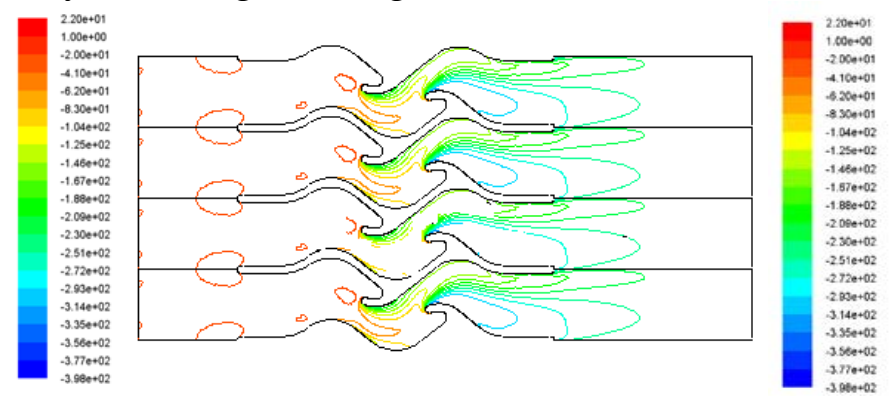

a) the inertial stage

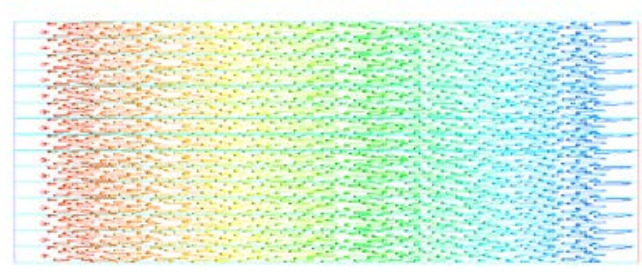

b) the multi-layer

Fig.5 Total pressure distribution of $(5 \mathrm{~m} / \mathrm{s})$ 
With the increasing inlet velocity, the total pressure drop of the inertial stage and the multi-layer gauze stage grows and its gradient becomes bigger. The 2-order polynomial pressure drop equation of the compound stage can be computed through the numerical simulation result : $\Delta P=$ $11.4447 v^{2}+16.2969 v$, where $\Delta P$ is the pressure drop and $v$ is the inlet velocity of the compound stage.

Comparing the above pressure drop equation with equation (8), porous media coefficient is derived by the equations as follows: fluid viscosity resistant coefficient $1 / \alpha=29.914 / \mu$; fluid inertial resistance coefficient $\mathrm{C}_{2}=26.334 / \rho$. One source term was added into the momentum equation accordingly, and the flowing characteristics of the filter were computed based on the porous medium model.

\section{Experiment validation.}

To validate the simulating results of the total pressure loss, a designed wind-tunnel experiment is installed. The experiment schematic of the resistance characteristic is designed and provided in figure 6. Resistant characteristic experiment with varying inlet velocities was carried out at the atmospheric pressure and ambient temperature (100600Pa, 300K).

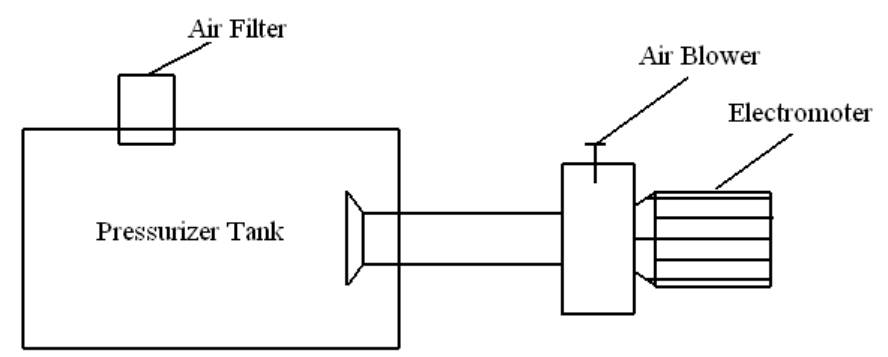

Fig.6Experimental schematic of filter

The primary measuring apparatuses contain the atmospheric pressure gauge, the indoor temperature and humidity meter, the TSI anemometer and the gas flow indicator. Because of the connection of the air filter and the pressurize tank, the sub-atmospheric pressure is equivalent to the total pressure loss value of the filter. Thus, the total pressure loss of the air filter could be measured by the TSI anemometer installed with the pressurize tank. The measured pressure loss line with varying inlet velocity was fitted in figure 7 to validate the simulation result.

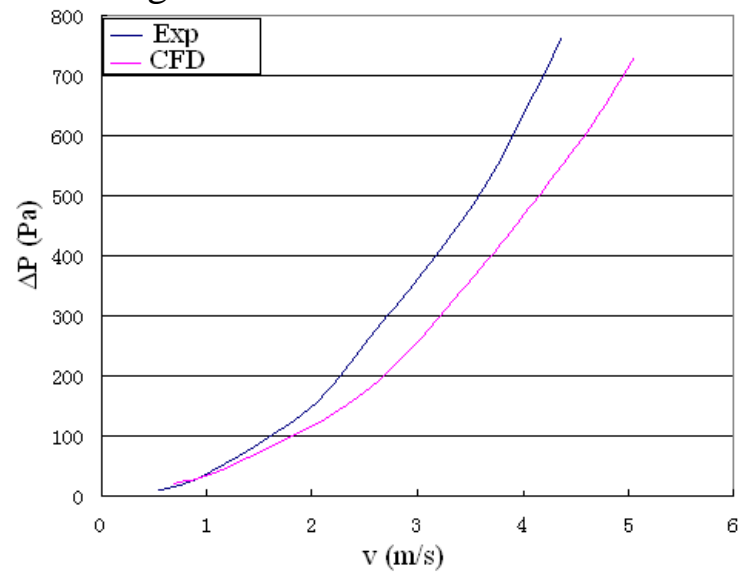

Fig.7 Resistance characteristic of the filter

As observed in the above figure, the total pressure loss increases with the raise of the inlet velocity of the air filter. Moreover, the distinction between numerical data and experimental data is limited and acceptable, which can be elided in engineering applications. The numerical resistance characteristic curve has a good agreement with the experimental one. What can be concluded is that porous media model is a feasible method to replace the filter element. 


\section{Conclusions}

Numerical simulation and experiment study were carried out to research the flowing field and the resistant characteristic of the offshore-platform air filter. Based on the established data, satisfactory results are obtained. The chief conclusions could be summed up as follows:

1) With 2D simplifications, a computational method for the inertial stage and the multi-layer gauze stage is proposed.

2) The resistant characteristic of the compound stage is obtained by CFD method based on simplified models. The quadratic polynomial of the resistance with velocity, the permeability $\alpha$ and the pressure jump coefficient $C_{2}$ were obtained.

3) By means of the porous media model substituting for the compound stage, simulation of the offshore-platform air filter of varying velocities using $k-\varepsilon$ model was conducted. Velocities, total pressure and turbulence energy of the filter were obtained and analyzed.

4) The numerical research of the air filter is validated by the designed resistant characteristic experiment. It shows that the compound stage replaced by porous media is feasible and acceptable.

\section{Acknowledgement}

The authors gratefully acknowledge the financial assistance from National Natural Science Foundation of China (Project 51309063 and 51409067), Specialized Research Fund for the Doctoral Program of Higher Education (Project: 20132304120012)

\section{References}

[1] B.E.Launder, D.B.Spalding (1972). Lectures in mathematical models of turbulence. Academic Press, London.

[2] Brekke, Olaf, Bakken. Lars.E (2004). Performance deterioration of intake air filters for gas turbines in offshore installations. Proceedings of ASME Turbo Expo2010: Power for Land, Sea and Air, Glasgow, 685-694.

[3] Chiara Galletti, Elisabetta Brunazzi, Leonardo Tognotti (2008). A numerical model for gas flow and droplet motion in wave-plate mist eliminators with drainage channels. Chemical Engineering Science. 30(1), 5639-5652.

[4] Coakley T.J. (1983). Turbulence methods for the compressible Navier-Stokes equations. AIAA-1983-1693.

[5] Elisabetta Brunazzi, Alessandro Paglianti (2001). Conventional and complex knitted mesh mist eliminators. Chemical Engineering Technology. 24(11), 1199-1204.

[6] Griffiths W.D., Boysan F. (1996). Computational fluid dynamics (CFD) and empirical modeling of the performance of a cyclone samplers. Journal of Aerosol Science. 27(2):281-304.

[7] Jones W.P., Launder B.E. (1973). The calculation of low Reynolds number phenomena with a two-equation model of turbulence. International Journal of the Heat Mass Transfer. 16:1119-1130

[8] Luan Yigang, Sun Haiou, Wang Song (2010). Performance study of a $\Omega$ type inertia gas-liquid separator. Journal of Engineering for Thermal Energy and Power. 28(3), 394-398.

[9] Rafee, R., Rahimzadeh, H. (2010). Performance evaluation of a curved type vane separator at different plate spacings in the range of $25 \mathrm{~mm}$ to $35 \mathrm{~mm}$ using numerical simulation. Iran Journal of Chemical Engineering. 29(3). 
[10] Sun Haiou, Sun Jun, Liu Xueyi (2002). Calculation of the resistance characteristics of a three-stage moisture separator for marine applications. Journal of Engineering for Thermal Energy \& Power. 17(4), 383-386.

[11] Sun Haiou, Zheng Hongtao and Liu Shunlong (2000). Numerical simulation of resistances of multilayer gauze stage in moisture eeparator. Journal of Harbin Engineering University. 21(4), 10-12.

[12] Souders, M.,Brown, G (1961). Design of fractionating columns. I. Entrainment and capacity. ARS Journal. 31(6), 1467.

[13] S.V.Patanker, D.B.Spalding (1972). A calcaulation processure for heat, Mass and momentum transfer in three-dimensional parabolic flows, International Journal of Heat Mass Transfer. 15(4), 1787-1806.

[14] Wu Jianghang, HAN Qingshu (1988). The theory, method and application of computational fluid dynamics. Science Press, Beijing.

[16] York, O.H.,Poppele, E.W (1954). Performance of wire-mesh demisters. Chemical Engineering and Processing. 50(8), 421-424. 